\title{
Opioid Use Disorder Treatment Initiation and Continuation: a Qualitative Study of Patients Who Received Addiction Consultation and Hospital-Based Providers
}

\author{
Susan L. Calcaterra, $M D, M P H^{1,2}{ }^{D}$, Steve Lockhart, $\mathrm{MPH}^{3}$, Catherine Callister, $\mathrm{MD}^{2}$, \\ Kaitlyn Hoover, $B A, M S^{4}$, and Ingrid A. Binswanger, $M D, M P H^{1,5,6,7}$
}

'Division of General Internal Medicine, University of Colorado, 8th Floor, Academic Office 1, Mailstop B180, 12631 E 17th Ave, Aurora, CO, USA; ${ }^{2}$ Division of Hospital Medicine, University of Colorado, Aurora, CO, USA; ${ }^{3}$ Adult and Child Consortium for Health Outcomes Research and Delivery Service, Univeristy of Colorado, School of Medicine and Children's Hospital Colorado, Aurora, CO, USA; ${ }^{4}$ Clinical Science Graduate Program, University of Colorado, Aurora, CO, USA; ${ }^{5}$ nstitute for Health Research, Kaiser Permanente Colorado, Aurora, CO, USA; ${ }^{6} \mathrm{Colorado}$ Permanente Medical Group, Aurora, CO, USA; ${ }^{7}$ Bernard J. Tyson School of Medicine, Pasadena, CA, USA.

\begin{abstract}
:
BACKGROUND: Hospitalizations related to opioid use disorder (OUD) are rising. Addiction consultation services (ACS) increasingly provide OUD treatment to hospitalized patients, but barriers to initiating and continuing medications for OUD remain. We examined facilitators and barriers to hospital-based OUD treatment initiation and continuation from the perspective of patients and healthcare workers in the context of an ACS.
\end{abstract}

METHODS: In this qualitative study, we sought input using key informant interviews and focus groups from patients who received care from an ACS during their hospitalization and from hospitalists, pharmacists, social workers, and nurses who work in the hospital setting. A multidisciplinary team coded and analyzed transcripts using a directed content analysis.

FINDINGS: We conducted 20 key informant interviews with patients, nine of whom were interviewed following hospital discharge and 12 of whom were interviewed during a rehospitalization. We completed six focus groups and eight key informant interviews with hospitalists and hospital-based medical staff $(n=62)$. Emergent themes related to hospital-based OUD treatment included the following: the benefit of an ACS to facilitate OUD treatment engagement; expanded use of methadone or buprenorphine to treat opioid withdrawal; the triad of hospitalization, self-efficacy, and easily accessible, patientcentered treatment motivates change in opioid use; adequate pain control and stabilization of mental health conditions among patients with OUD contributed to opioid agonist therapy (OAT) continuation; and stable housing and social support are prerequisites for OAT uptake and continuation.

CONCLUSION: Modifiable factors which facilitate hospital-based OUD treatment initiation and continuation include availability of in-hospital addiction expertise to offer easily accessible, patient-centered treatment and the use of methadone or buprenorphine to manage opioid withdrawal. Further research and public policy efforts are urgently needed to address reported barriers to hospitalbased OUD treatment initiation and continuation which

Received August 7, 2021

Accepted November 23, 2021

Published online January 3, 2022 include unstable housing, poorly controlled chronic medical and mental illness, and lack of social support.

KEY WORDS: qualitative; addiction; opioid use disorder; opioid agonist; buprenorphine; methadone.

J Gen Intern Med 37(11):2786-94

DOI: $10.1007 / \mathrm{s} 11606-021-07305-3$

(C) The Author(s) under exclusive licence to Society of General Internal Medicine 2021

\section{INTRODUCTION}

Hospitalizations related to opioid use and opioid overdose quadrupled in the past three decades. ${ }^{1}$ Medical complications of injection drug use, including solid organ infections, may require weeks of intravenous antibiotics to ensure bacterial clearance. ${ }^{2}$ People who inject drugs often remain hospitalized to complete antibiotic treatment which may be challenging for those who lack social support or coping skills. ${ }^{3,4}$ Consequently, patients with substance use disorders and healthcare providers report difficult interactions related to in-hospital substance use, poorly controlled withdrawal, patients leaving prior to treatment completion, and in-hospital overdose deaths. $^{5-8}$ To address increasing opioid-related hospitalizations, some hospitals have implemented addiction consultation services (ACS). ${ }^{9}$ ACS meet with hospitalized patients to discuss their substance use, offer treatment, and link patients to post-discharge addiction treatment. ${ }^{10-13}$ Provision of hospitalbased addiction treatment is associated with reduced rehospitalization and increased post-discharge treatment engagement. $^{14,15}$

Population-level data has demonstrated that receipt of opioid agonist therapy (OAT), including buprenorphine or methadone, following an overdose event was associated with a $50 \%$ mortality reduction over 12 months compared to no treatment receipt. ${ }^{16}$ In the outpatient setting, patient-reported barriers to OAT initiation and continuation include negative perceptions of treatment by family, friends, or healthcare providers, difficulty accessing treatment, and lack of 
motivation to remain in treatment. ${ }^{17}$ The ACS is a novel intervention that is becoming increasingly implemented in hospitals in North America. ${ }^{9}, 10,18,19$ As hospitals expand access to ACS, we identified facilitators and barriers to hospital-based opioid use disorder (OUD) treatment initiation and continuation. We included the perspective of patients who received care from an ACS during hospitalization and from hospitalists and hospital-based nurses, social workers, and pharmacists who cared for hospitalized patients with OUD. This study was conducted to inform efforts to expand patientcentered, hospital-based OUD treatment, with a focus on treatment uptake and retention.

\section{METHODS}

\section{Study Design}

We conducted key informant interviews and focus groups to understand facilitators and barriers to hospital-based OUD treatment initiation and continuation from the perspective of patients with OUD and from hospital-based providers (hospitalists, nurses, social workers, and pharmacists). The University of Colorado Institutional Review Board determined this project to be exempt and not human subject research because we did not collect any participant identifiers (Protocol \# 19-0336). Three theoretical frameworks informed study design and analysis: the Health Belief Model $^{20,}{ }^{21}$; the Knowledge, Attitudes, and Practices Framework ${ }^{22}$; and the Donabedian Framework. ${ }^{23}$ The analysis was not preregistered and the results should be considered exploratory. Data collection, coding, and analysis closely followed the consolidated criteria for qualitative research (COREQ) (checklist available upon request). ${ }^{24}$

\section{Study Settings and Participants}

We recruited patients who received care from a hospital-based ACS at a university hospital. ACS team members (SLC and $\mathrm{CC}$ ) recruited patients for study inclusion by calling them to inquire about their interest in study participation with a brief description of the study purpose. At the time of the interview, participants either were discharged from their initial hospitalization or had been re-hospitalized. We interviewed participants one-on-one over the telephone or in their private hospital room. Interviews lasted approximately $1 \mathrm{~h}$, and we provided a gift card as compensation for participation.

We recruited providers from two locations, a large public hospital and a university hospital in Denver, Colorado. Both hospitals employ a weekday ACS. We used a purposeful sampling strategy for recruitment. We contacted the Director of Services for Hospital Medicine and team leaders for nursing, social workers, and pharmacy staff who distributed an email solicitation for focus group participation with a brief description of the study purpose. Focus group participants were segmented into practice type: hospitalists, pharmacists, and nurses with social workers because we anticipated that homogenous groups would allow for more free-flowing conversations. ${ }^{25}$ One-hour focus group sessions were held in a hospital conference room at noontime with lunch provided as compensation for participation. Due to low focus-group participation by nurses based on COVID-19-related scheduling conflicts, we also conducted one-on-one key-informant interviews with nurses over the telephone at their convenience. These interviews lasted approximately $45 \mathrm{~min}$, and gift cards were provided for compensation. Recruitment for both groups continued until it appeared that data saturation was reached. ${ }^{26}$ Study participants were informed that interviews were confidential and provided verbal consent prior to data collection.

Study Team and Key Informant Interview and Focus Group Guide Content and Structure

Our multidisciplinary team was comprised of two addiction-trained clinician researchers (SLC, IAB), a hospitalist physician (CC), a trained qualitative analyst (SL), and a master's student in clinical science $(\mathrm{KH})$.

\section{Patient Key Informant Interviews}

SLC and SL developed a patient interview guide to elicit information regarding their hospitalization experience and whether it impacted their substance use, their experiences with the medical team and the ACS, their perceptions of the hospital discharge process, and the ease of linkage to addiction treatment post-discharge. Among rehospitalized patients, we inquired if the ACS could have provided services to prevent rehospitalization. The interview guide was informed by the authors' experience in clinical practice (SLC and CC) and by a literature review of patients' perspectives regarding addiction treatment (Appendix A). ${ }^{6}$ 17, 27,28 Interviews were conducted by two physicians (SLC and CC), both of whom work on their institution's ACS. SLC and CC previously provided medical treatment to some study participants, but not during the study period.

\section{Hospital-Based Provider Focus Groups and Key Informant Interviews}

SLC and SL developed three provider-specific focus group guides (hospitalists, pharmacists, and nurses plus social workers; Appendix B-D) informed by two theoretical frameworks: the Knowledge, Attitudes, and Practices Framework, which identifies barriers to physician adherence to practice guidelines in relation to behavior change, and the Donabedian Framework for the Evaluation of the Quality of Care, which includes measures to identify structures, process, and outcome measures of care provided by healthcare organizations. ${ }^{22,} 23$ The primary facilitator of each focus group was trained in qualitative research methods (SL) and was joined by $\mathrm{KH}$. $\mathrm{JH}, \mathrm{SC}$, and $\mathrm{CC}$ participated as co-facilitators and recorded field notes. Additional key informant interviews with nurses were conducted by SLC and SL using a modified focus group guide. 


\section{Data Analysis}

Focus groups and key informant interviews were recorded, transcribed, and entered into ATLAS.ti data management software. No identifiers were collected or recorded. We employed both a deductive and inductive approach, based on a directed content analysis, to analyze our data. ${ }^{29-31}$ A deductive approach was used to transcript text to predefined codes and categories informed by the literature, ${ }^{28,32,33}$ prior knowledge, our interview guides, and explanatory frameworks. Explanatory frameworks used to inform a priori code development included the Health Belief Model, ${ }^{20}$ a model developed to explain and predict health-related behaviors, used with the patient transcripts, and the Knowledge, Attitudes, and Practices Framework ${ }^{22}$ and the Donabedian Framework ${ }^{23}$ used with the hospital-based provider transcripts. An inductive approach was used to identify emergent codes or categories which included new ideas relevant to our research question. ${ }^{34}$ Two separate codebooks were created for patient and hospitalbased provider transcripts.

The coding team (SLC, SL, KH, CC) independently coded three patient transcripts and three focus group transcripts by assigning the predefined codes to the text and by creating new codes that emerged from the data. The team met to review code agreement and reconcile any differences until consensus was reached. Next, the team coded the remaining transcripts while meeting frequently to iteratively refine the codes and definitions. All transcripts were double coded. Individual codes were categorized into larger codes to facilitate analysis. The team met regularly to discuss emerging themes and relevancy to hospital-based OUD treatment uptake and continuation. Themes were subsequently presented and discussed with experienced researchers and hospitalbased providers who work closely with the OUD patient population. Once we reached consensus on themes for the patient and provider datasets, we used a constant comparative method and reflexive team-based analysis to examine commonalities and differences across each dataset. We highlighted themes reflective of the patient experience and themes that overlapped between the patient and hospital-based provider experience.

\section{RESULTS}

Between February 2020 and June 2021, we recruited and conducted 20 interviews with patients who interacted with the ACS during hospitalization. Of these, nine patients were interviewed over the telephone and 11 were in-hospital interviews. Seventy-five percent of patients reported their ethnicity as non-Hispanic White $(n=15)$. All patients reported opioid use, $11(55 \%)$ reported opioid and methamphetamine use, and two $(10 \%)$ reported opioid and alcohol use (Table 1). Between November 2019 and February 2020, we recruited
Table 1 Participant Characteristics $(n=82)$

\begin{tabular}{ll}
\hline \hline Patient data $(\boldsymbol{n}=\mathbf{2 0})$ & $\boldsymbol{n ( \% )}$ \\
\hline Gender & \\
$\quad$ Female & $7(35)$ \\
Male & $13(65)$ \\
Ethnicity & $15(75)$ \\
White, non-Hispanic & $5(25)$ \\
Hispanic & $9(45)$ \\
Interviewed outpatient & $11(55)$ \\
Interviewed in-hospital & \\
Reported substance(s) used ${ }^{\dagger}$ & $7(35)$ \\
Opioids alone & $11(55)$ \\
Opioids and methamphetamine & $2(10)$ \\
Opioids and alcohol & $\boldsymbol{n}(\mathbf{\%})$ \\
Healthcare provider data (n=62) & \\
Gender & $46(75)$ \\
Female & $16(25)$ \\
Male & $19(31)$ \\
Professional role & $18(30)$ \\
Hospitalist physician & $13(21)$ \\
Pharmacist & $11(18)$ \\
Nurse & \\
Social worker & $20(33)$ \\
Years working in current job type & $17(28)$ \\
<5 & $16(26)$ \\
6-10 & $8(13)$ \\
11-15 &
\end{tabular}

*Re-hospitalized at time of interview

†No reported regular use of cocaine or benzodiazepines

$t n=62$ participants; one participant did not complete a survey

$\$ 8 / 13$ nurses participated in individual interviews due to COVID-19related scheduling conflicts

and conducted six in-person focus groups with hospitalbased providers for an average of nine participants per group. Hospitalists $(n=19,31 \%)$, pharmacists $(n=18$, $30 \%)$, nurses $(n=13,21 \%)$, and social workers $(n=$ $11,18 \%)$ were represented. We conducted telephone interviews with nurses at both hospitals $(n=8)$. The majority of participants were women $(n=46,75 \%)$ (Table 1). From this broad range of perspectives, we identified five emergent themes as they pertained to hospital-based OUD treatment initiation and continuation including: (1) the role of an ACS to address OUD and facilitate treatment engagement; (2) expanded use of OAT to treat opioid withdrawal and to improve patient-provider interactions; (3) the triad of hospitalization, self-efficacy, and patient-centered treatment (defined as low-threshold treatment) motivated opioid use behavior change ${ }^{35}$; (4) the necessity of pain control and mental health stability for OAT continuation; and (5) the significance of stable housing and social support for OAT initiation and continuation. Themes 1, 2, and 5 included perspectives from patients and hospital-based providers. Themes 3 and 4 were limited to patient perspectives (Fig. 1).

\section{Key Themes}

Theme 1: Benefits of an ACS to Address OUD and to Facilitate Treatment Engagement. Patients and hospitalbased providers perceived the role of the ACS as reducing 


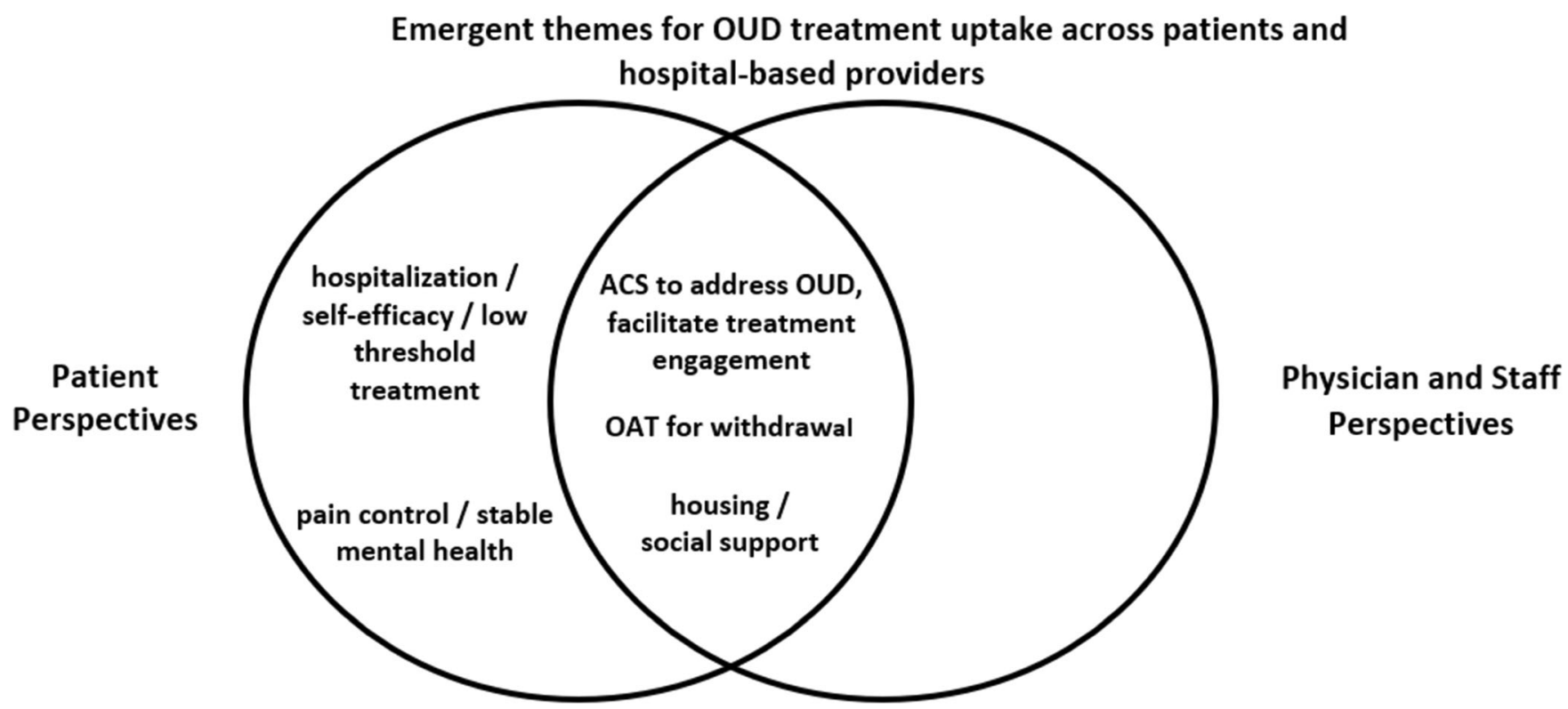

Figure 1 Emergent themes for OUD treatment uptake across patients and hospital-based providers.

shame, providing information, and supporting OUD treatment engagement and follow-up. Patients reported that their interactions with ACS contributed to feelings of greater self-worth and improved their understanding of treatment options, including medications to treat OUD. One patient described their experiences meeting with the ACS:

"They [ACS] made me feel like I am a good person. I am not a criminal. I am worth having a life. I am worth them taking their time to work on me. That made me feel so good." (Patient 7)

Another person discussed their shame and concerns with using buprenorphine with the ACS:

"I have a lot more positive take on it. Before, I felt really ashamed taking buprenorphine 'cause, people always assume you're a heroin addict, so people made me feel ashamed to take it. When I talked to them, they were really understanding, so it made me feel a lot better. They were very informative, very nice, very caring." (Patient 2)

Hospital-based providers identified that the ACS allowed for dedicated time and resources to spend with patients to discuss personal goals as a way to provide patient-centered care:

"I definitely think, with addiction medicine being able address the addiction piece and what kind of treatment that they might want after, and what other factors might come after that, just having the resources and someone that's able to focus in and help the patient in whatever way they want, that they see themselves succeeding, it has been helpful.” (Focus group 6, social worker)
Patients and hospital-based providers appreciated that the ACS linked patients to addiction treatment following discharge. Patients appreciated the removal of barriers to obtain treatment following hospitalization:

"They [ACS] didn't just hand me a packet and say, "All right, here's your information, good luck." They [ACS] actually followed through. That helps because it takes some of the pressure off 'cause, sometimes it's overwhelming. It's like, "Okay, I've got all these phone calls to make. I've got all this stuff to do." Having me already set up with something when I got out [of the hospital] was immensely helpful." (Patient 6)

Hospital-based providers reported that treatment initiation with care linkage contributed to a sense of providing highquality care to support patients in recovery, even following discharge:

"I think they're [the ACS] are able to hook them up with our methadone services easily so that, as they leave the hospital, they can follow up directly with our methadone clinic." (Nurse interview 3)

Theme 2: Expanded Use of Opioid Agonists to Treat Opioid Withdrawal Improved Patient-Provider Interactions.. Patients and hospital-based providers appreciated the use of OAT in the hospital to reduce opioid withdrawal symptoms and improve patient-provider interactions. One patient described feeling greater relief from opioid withdrawal when prescribed OAT, in contrast to short-acting opioids, traditionally used to manage opioid withdrawal. 
The use of methadone allowed him to remain in the hospital to receive necessary medical treatment.

"Methadone helped. Dilaudid and oxycodone just don't work [for treating opioid withdrawal]. It's gotta be heroin or methadone. I stayed longer than I would have had I not been on [methadone] because the withdrawals were really bad." (Patient 20)

Hospitalists noted that when they prescribed OAT to manage withdrawal, their interactions with patients improved.

“They're just so challenging [patients experiencing opioid withdrawal]. Once you get them on Suboxone, they're much more engaged and receptive. And I go, "Oh, this is who you are." But when you are withdrawing, you're not yourself." (Focus group 4, hospitalist)

Some pharmacists noted that as physicians used more methadone and buprenorphine, they could benefit from a better understanding of the medications, the legality of their use, and indications for their use.

"As their use is increasing [methadone and buprenorphine], I think the education on the pharmacist side needs to match up to that of the physicians. We need a better understanding of these medications because we are seeing it more in practice." (Focus group 2, pharmacist)

Theme 3: The Triad of Hospitalization, Self-Efficacy, and Low-Threshold Treatment Motivated Change in Opioid Use Behaviors. Some patients described hospitalization as a motivator to make a change in their opioid use, especially when accompanied by a preexisting desire to make a change in their lifestyle or use behaviors.

"I thought I was dying, not giving life. It turns out I was growing a human being inside of me. They [the ACS] gave me the correct dose of Suboxone, they found me programs and resources. I didn't want my baby being born on heroin going through withdrawal." (Patient 6)

For some patients, hospitalization removed barriers to make changes in their opioid use.

"When this opportunity came up about Suboxone when I was in the hospital, I was a little hesitant, but I said, 'I'm gonna take the leap and start takin' this. Maybe it will change things."' (Patient 4)

For others, the thought of changing their opioid use was inconceivable, often expressing hopelessness and helplessness over their addiction.
"The methadone clinic didn't matter to me. Once I put the heroin in me, I don't care about that. I'm gonna use when I get out 'cause I don't wanna be sick.” (Patient 17)

Theme 4: Adequate Pain Control and Stabilization of Mental Health Conditions Among Patients with OUD Contributed to OAT Continuation.. Chronic pain and mental illness were closely linked with substance use behaviors. Some patients used alcohol and opioid pills to manage chronic pain, while others used heroin. For these patients, the end result contributed to their hospitalization. For one patient, in-hospital buprenorphine initiation allowed for improved pain control and stabilization of substance use.

"When I went in the hospital, I was out of it [alcohol withdrawal seizure], and then when I came outta the hospital, he [primary care provider] didn't prescribe oxycodone to me for a month. I was just miserable in pain so I went back to drinkin'. Ever since they prescribed Suboxone to me, it's just been more of an even, steady little bit pain, very livable, very manageable, like it's just a different world." (Patient 4)

For some patients, initiation of methadone or buprenorphine was insufficient to continue OAT following discharge. One barrier to OAT continuation was persistent pain which was only controlled by heroin use.

"The heroin was cheaper [than buprenorphine]. I didn't get high or anything, but I felt the pain go away. It's not about getting high. It's about getting the pain gone so I can function normally." (Patient 18)

While the ACS initiated medications for OUD, prescribed antidepressant or anxiolytic medications, and made referrals to behavioral health clinics, this was not sufficient for treatment continuation among some patients with serious mental illness. One patient stated:

"I'm screwed up in the head so I'm gonna end up in the hospital all the time anyway." (Patient 11)

Another said:

"I have PTSD. My drug abuse helps cover that pain, emotional and physical pain." (Patient 19)

There was little the ACS could do during hospitalization to support these patients following hospital discharge beyond linking them to community resources.

Theme 5: Stable Housing and Social Support Are Prerequisites for Continued OAT Engagement. Patients described the importance of stable housing and social 
support to continue OAT following hospital discharge, which included daily visits to an opioid treatment program or regular visits to a buprenorphine-waivered prescriber. A lack of resources was often cited as a barrier for OAT continuation.

"I would go back [to substance use] 'cause I have nobody, no resources, no help, no housing. I'm disabled. I have nobody helping me." (Patient 10)

Patients described the importance of housing and social support for their change in opioid use:

"Having a place to go home to, and a bed. You're not gonna have to think about, 'Where's the next place I'm gonna sleep tonight?", I think that's such a key part in sobriety." (Patient 6)

Hospital-based providers recognized the importance of stable housing and social support for OUD treatment continuation which could be facilitated by programs offering housing and support services.

"Hooking them up with a program that could lead to housing [could reduce opioid use]. Homelessness is a great contributor to alcohol and drug use. You can't really start to focus on quitting drugs if you don't have anywhere to live." (Focus group 3, social worker)

\section{DISCUSSION}

Patients with OUD and hospital-based providers identified several facilitators and barriers to hospital-based OUD treatment uptake and continuation. Perceived facilitators included the presence of an ACS to discuss and initiate OUD treatment, increased use of OAT to improve opioid withdrawal and to improve patient-provider interactions, and provision of easily accessible, patient-centered OAT in the hospital. Perceived barriers to OUD treatment continuation included uncontrolled pain, active mental illness, and a lack of stable housing and social support.

Patients with substance use disorders report experiencing stigma in the healthcare system, which is a barrier to OUD treatment uptake. ${ }^{17,36}$ In our study, several patients described respectful and informative interactions with the ACS. For some patients, discussing treatment options with the ACS reduced their own personal stigma with OAT. Hospitalbased providers appreciated having the ACS available to discuss treatment options for their patients and to facilitate addiction treatment linkage following discharge. With the presence of an ACS, non-addiction providers are exposed to effective medications and behavioral interventions for OUD, thus normalizing OUD and the medications used to manage it. Previous work demonstrated that "treatment works" messaging may mitigate addiction-related stigma. ${ }^{37,} 38$ As more hospitals implement ACS to provide evidenced-based treatment, the stigma experienced by hospitalized patients with OUD may diminish. In the meantime, hospitals should support training and education of hospital-based providers to extend their knowledge, skills, and self-efficacy to best care for hospitalized patients with OUD. ${ }^{39}$

Patients and hospital-based providers identified uncontrolled pain, active mental illness, and a lack of housing and social support as barriers to OUD treatment continuation following hospital discharge. Chronic pain management in the setting of OUD is complicated by opioid-induced hyperalgesia, high rates of co-occurring psychiatric disorders, and increased risk of relapse compared to patients with OUD alone. ${ }^{40-42}$ Chronic mental illness is challenging to manage when people are actively using substances, when they lack stable housing, and when they are unable to access routine mental health care. ${ }^{43-48}$ Hospitals are increasingly partnering with community organizations to provide housing to people with severe, chronic health problems. ${ }^{49}$ Additionally, many hospitals provide medical respite to people experiencing homelessness who are too ill to recover on the streets, but are not ill enough to remain in the hospital. ${ }^{50}$ Hospitalized patients with severe OUD often experience many of these barriers, leading to frequent re-hospitalizations and increased mortality. ${ }^{51,52}$ For people experiencing severe mental illness, an assertive community treatment model, which offers around-the-clock, customized, community-based services to people in their homes, has been associated with a reduction in homelessness ${ }^{53}$ and in substance use compared to standard case management. ${ }^{54,55} \mathrm{~A}$ combination of assertive community treatment, low-threshold OAT, and stable housing may improve utilization of healthcare services and mortality among this vulnerable group of people. ${ }^{16,53,56}$

The use of OAT to manage opioid withdrawal combined with readily accessible OUD treatment, provision of patientcentered care, and care linkage were identified as facilitators of OUD treatment uptake. The presence of an ACS facilitates education and awareness of these evidence-based practices for OUD treatment; however, an ACS is not absolutely necessary for clinical practice uptake, implementation, and dissemination. In hospitals where ACS implementation may not be feasible or necessary, successful models targeted at OUD treatment exist. Key aspects of these programs include a project champion to advocate for hospitalized patients by correctly diagnosing OUD, in-hospital buprenorphine initiation with continuation at discharge, co-prescribing of naloxone for overdose reversal, and linkage to addiction treatment upon discharge. ${ }^{57-60}$ Hospital-based clinicians and academic societies advocate for their colleagues to incorporate OUD treatment into their daily practice to "drive societal change by removing barriers for patients and mitigating the stigma against being an $\mathrm{X}$-waivered clinician., ${ }^{\text {} 60,61}$ Calls to remove restrictions on buprenorphine prescribing led to an exemption from the $\mathrm{X}$-wavier training requirements and certification to psychosocial services for clinicians who prescribe 
buprenorphine to 30 or less patients at any given time. ${ }^{62-65}$ Hospitals should support their staff to develop innovative education and training strategies to expand hospital-based OUD treatment initiation and care linkage. Insurers should provide higher reimbursement for provision of OUD services to incentive providers to incorporate this work into their clinical practice. Future work should evaluate if such programs reduce growing costs associated with opioid-related hospitalizations and help patients achieve their personal goals related to opioid use, including incorporating harm reduction strategies into their opioid use, initiating OAT when indicated, and/ or engaging patients in behavioral health treatment. ${ }^{66}$

\section{LIMITATIONS}

Our qualitative findings may not reflect the experiences of all hospitalized patients with OUD and hospital-based providers outside of these hospital settings. ${ }^{67}$ Patient interviews were conducted at one hospital. Additional patient perspectives are needed for greater generalizability and to extend these findings. Patient participants may have previously received care from a study team member which could have influenced their study participation or responses. We attempted to mitigate this by ensuring that team members conducting the interviews were not part of the patient's medical team and by reassuring participants that their interviews were anonymous and voluntary. It is likely that our past relationships with some participants improved recruitment due to mutual appreciation, trust, and respect. Findings may be influenced by the perspectives of investigators during the collection and data analysis. We assembled a multidisciplinary team and used a team-based, iterative process with triangulation and reflexivity to employ a rigorous approach to our study questions.

\section{CONCLUSION}

Our study identified important facilitators of OUD treatment uptake and continuation that can be implemented in hospitals, including expanded access to in-hospital addiction expertise, greater use of OAT to manage opioid withdrawal, and provision of low-threshold OUD treatment. While various models exist to expand hospital-based addiction treatment, many require a project champion with knowledge and clinical experience managing OUD. ${ }^{14,}{ }^{57}$ Currently, multisite studies are underway to measure the effectiveness and reach of hospitalbased ACS on post-discharge treatment initiation and engagement. ${ }^{68}$ Further research and public policy efforts are needed to address barriers to OUD treatment uptake raised by study participants regarding lack of stable housing, chronic mental and medical illness, and lack of social support.

Corresponding Author: Susan L. Calcaterra, MD, MPH; Division of General Internal Medicine, University of Colorado, 8th Floor, Academic Office 1, Mailstop B180, 12631 E 17th Ave, Aurora, CO 80045, USA (e-mail: susan.calcaterra@cuanschutz.edu).
Supplementary Information The online version contains supplementary material available at https://doi.org/10.1007/s11606-02107305-3.

Funding Dr. Calcaterra is supported by the National Institute on Drug Abuse (NIDA), National Institutes of Health (NIH), grant award number K08DA049905. Dr. Binswanger is supported by grants R01DA042059 and R01DA047537. The views and opinions expressed in this article are those of the authors and do not necessarily reflect those of the NIH.

\section{Declarations:}

Conflict of Interest: The authors declare no competing interests.

\section{REFERENCES}

1. Peterson C, Xu L, Florence C, Mack KA. Opioid-related US hospital discharges by type, 1993-2016. J Subst Abus Treat. 2019;103:9-13.

2. Baddour LM, Wilson WR, Bayer AS, Fowler VG, Jr., Tleyjeh IM, Rybak MJ, et al. Infective Endocarditis in Adults: Diagnosis, Antimicrobial Therapy, and Management of Complications: A Scientific Statement for Healthcare Professionals From the American Heart Association. Circulation. 2015;132(15): 1435-86.

3. Suzuki J, Johnson J, Montgomery M, Hayden M, Price C. Outpatient Parenteral Antimicrobial Therapy Among People Who Inject Drugs: A Review of the Literature. Open Forum Infect Dis. 2018;5(9):ofy 194.

4. Suzuki J, Robinson D, Mosquera M, Solomon DA, Montgomery MW, Price $\mathrm{CD}$, et al. Impact of Medications for Opioid Use Disorder on Discharge Against Medical Advice Among People Who Inject Drugs Hospitalized for Infective Endocarditis. Am J Addict. 2020;29(2):155-9.

5. Fanucchi LC, Lofwall MR, Nuzzo PA, Walsh SL. In-hospital illicit drug use, substance use disorders, and acceptance of residential treatment in a prospective pilot needs assessment of hospitalized adults with severe infections from injecting drugs. J Subst Abus Treat. 2018;92:64-9.

6. Velez CM, Nicolaidis C, Korthuis PT, Englander H. "It's been an experience, a life learning experience": a qualitative study of hospitalized patients with substance use disorders. J Gen Intern Med. 2017;32(3):296-303.

7. Roy PJ, Saitz R. The Cost of a Cigarette: Opportunities to Engage Hospitalized Patients With Opioid Use Disorder. J Addict Med. 2021;15(1):13-4.

8. Calcaterra SL, Drabkin AD, Leslie SE, Doyle R, Koester S, Frank JW, et al. The hospitalist perspective on opioid prescribing: A qualitative analysis. J Hosp Med. 2016;11(8):536-42.

9. Priest KC, Englander H, McCarty D. "Now hospital leaders are paying attention": a qualitative study of internal and external factors influencing addiction consult services. J Subst Abus Treat. 2020;110:59-65.

10. Priest KC, McCarty D. The Role of the Hospital in the 21 st Century Opioid Overdose Epidemic: The Addiction Medicine Consult Service. J Addict Med. 2019;13(2): 104

11. Weinstein ZM, Wakeman SE, Nolan S. Inpatient addiction consult service: expertise for hospitalized patients with complex addiction problems. Med Clin. 2018;102(4):587-601.

12. Nenninger K, Carwile J, Fellers J, Thakarar K. Characteristics of Inpatients with Opioid Use Disorder Seen by "IMAT" Consult Service from 7/2016-6/2017. 2019. 2019). Maine Medical Center. 681. https:// knowledgeconnection.mainehealth.org/mmc/681

13. Thompson HM, Hill K, Jadhav R, Webb TA, Pollack M, Karnik N. The Substance Use Intervention Team: A Preliminary Analysis of a Population-level Strategy to Address the Opioid Crisis at an Academic Health Center. J Addict Med. 2019;13(6):460-463.

14. Englander H, Dobbertin K, Lind BK, Nicolaidis C, Graven P, Dorfman C, et al. Inpatient addiction medicine consultation and post-hospital substance use disorder treatment engagement: a propensity-matched analysis. J Gen Intern Med. 2019;34(12):2796-803.

15. Wakeman SE, Kane M, Powell E, Howard S, Shaw C, Regan S. Impact of inpatient addiction consultation on hospital readmission. J Gen Intern Med. 2021;36:2161-2163. https://doi.org/10.1007/s11606-020-05966-0 
16. Larochelle MR, Bernson D, Land T, Stopka TJ, Wang N, Xuan Z, et al. Medication for opioid use disorder after nonfatal opioid overdose and association with mortality: a cohort study. Ann Intern Med. 2018;169(3): 137-45.

17. Hall NY, Le L, Majmudar I, Mihalopoulos C. Barriers to accessing opioid substitution treatment for Opioid Use Disorder: A systematic review from the client perspective. Drug Alcohol Depend. 2021;221:108651.

18. Braithwaite V, Ti L, Fairbairn N, Ahamad K, McLean M, Harrison S, et al. Building a hospital-based addiction medicine consultation service in Vancouver, Canada: the path taken and lessons learned. Addiction. 2021;116(7):1892-1900.

19. Hyshka E, Morris H, Anderson-Baron J, Nixon L, Dong K, Salvalaggio G. Patient perspectives on a harm reduction-oriented addiction medicine consultation team implemented in a large acute care hospital. Drug Alcohol Depend. 2019;204:107523.

20. Janz NK, Becker MH. The Health Belief Model: A Decade Later. Health Educ Q. 1984;11(1):1-47.

21. Glanz K, Rimer BK, Viswanath K. Health behavior and health education: theory, research, and practice: John Wiley \& Sons; 2008.

22. Cabana MD, Rand CS, Powe NR, Wu AW, Wilson MH, Abboud P-AC, et al. Why don't physicians follow clinical practice guidelines?: A framework for improvement. JAMA. 1999;282(15):1458-65.

23. Donabedian A. Evaluating the quality of medical care. Milbank Mem Fund Q. 1966;44(3):166-206.

24. Tong A, Sainsbury P, Craig J. Consolidated criteria for reporting qualitative research (COREQ): a 32-item checklist for interviews and focus groups. Int J Qual Health Care. 2007; 19(6):349-57.

25. Morgan DL. Focus Groups as Qualitative Research. 2nd Edition ed. Thousand Oaks, California: SAGE University Paper; 1997.

26. Guest G, Bunce A, Johnson L. How many interviews are enough? An experiment with data saturation and variability. Field Methods 2006; 18(1):59-82.

27. Englander H, Collins D, Perry SP, Rabinowitz M, Phoutrides E, Nicolaidis C. "We've Learned It's a Medical Illness, Not a Moral Choice": Qualitative Study of the Effects of a Multicomponent Addiction Intervention on Hospital Providers' Attitudes and Experiences. J Hosp Med. 2018;13(11):752-8.

28. Finlay AK, Morse E, Stimmel M, Taylor E, Timko C, Harris AH, et al. Barriers to Medications for Opioid Use Disorder Among Veterans Involved in the Legal System: a Qualitative Study. J Gen Intern Med. 2020;35(9):2529-36.

29. Fereday J, Muir-Cochrane E. Demonstrating rigor using thematic analysis: A hybrid approach of inductive and deductive coding and theme development. Int J Qual Methods. 2006;5(1):80-92.

30. Elo S, Kyngäs H. The qualitative content analysis process. J Adv Nurs. 2008;62(1):107-15.

31. Hsieh H-F, Shannon SE. Three approaches to qualitative content analysis. Qual Health Res. 2005;15(9):1277-88.

32. Rawson RA, Rieckmann T, Cousins S, McCann M, Pearce R. Patient perceptions of treatment with medication treatment for opioid use disorder (MOUD) in the Vermont hub-and-spoke system. Prev Med. 2019; 128: 105785 .

33. Snow RL, Simon RE, Jack HE, Oller D, Kehoe L, Wakeman SE. Patient experiences with a transitional, low-threshold clinic for the treatment of substance use disorder: a qualitative study of a bridge clinic. J Subst Abus Treat. 2019;107:1-7.

34. Thomas DR. "A general inductive approach for analyzing qualitative evaluation data." Am J Eval. 2006;27(2):237-246.

35. Jakubowski A, Fox A. Defining Low-threshold Buprenorphine Treatment J Addict Med. 2020;14(2):95-8.

36. Van Boekel LC, Brouwers EP, Van Weeghel J, Garretsen HF. Stigma among health professionals towards patients with substance use disorders and its consequences for healthcare delivery: systematic review. Drug Alcohol Depend. 2013;131(1-2):23-35.

37. McGinty EE, Goldman HH, Pescosolido B, Barry CL. Portraying mental illness and drug addiction as treatable health conditions: effects of a randomized experiment on stigma and discrimination. Soc Sci Med. 2015; 126:73-85.

38. McGinty EE, Barry CL. Stigma reduction to combat the addiction crisis—developing an evidence base. N Engl J Med. 2020;382(14):1291-2.

39. Howard V, Holmshaw J. Inpatient staff perceptions in providing care to individuals with co-occurring mental health problems and illicit substance use. J Psychiatr Ment Health Nurs. 2010;17(10):862-72.
40. Marion Lee M, Sanford Silverman M, Hans Hansen M, Vikram Patel M, Laxmaiah Manchikanti M. A comprehensive review of opioid-induced hyperalgesia. Pain Physician. 2011;14:145-61.

41. Caldeiro RM, Malte CA, Calsyn DA, Baer JS, Nichol P, Kivlahan DR, et al. The association of persistent pain with out-patient addiction treatment outcomes and service utilization. Addiction. 2008;103(12): 1996-2005.

42. Barry DT, Cutter CJ, Beitel M, Kerns RD, Liong C, Schottenfeld RS. Psychiatric disorders among patients seeking treatment for co-occurring chronic pain and opioid use disorder. J Clin Psychiatry. 2016;77(10):1413-9.

43. Drake RE, Mueser KT, Brunette MF. Management of persons with cooccurring severe mental illness and substance use disorder: program implications. World Psychiatry. 2007;6(3):131-6.

44. RachBeisel J, Scott J, Dixon L. Co-occurring severe mental illness and substance use disorders: a review of recent research. Psychiatr Serv 1999;50(11):1427-34.

45. Kirst M, Zerger S, Misir V, Hwang S, Stergiopoulos V. The impact of a Housing First randomized controlled trial on substance use problems among homeless individuals with mental illness. Drug Alcohol Depend. 2015;146:24-9.

46. Patterson ML, Somers JM, Moniruzzaman A. Prolonged and persistent homelessness: multivariable analyses in a cohort experiencing current homelessness and mental illness in Vancouver, British Columbia. Ment Health Subst Use. 2012;5(2):85-101.

47. Smith TE, Sederer LI. A new kind of homelessness for individuals with serious mental illness? The need for a" mental health home". Psychiatr Serv. 2009;60(4):528-33.

48. Schanzer B, Dominguez B, Shrout PE, Caton CL. Homelessness, health status, and health care use. Am J Public Health. 2007;97(3):464-9.

49. Kuehn BM. Hospitals Turn to Housing to Help Homeless Patients. JAMA. 2019;321(9):822-4.

50. Kertesz SG, Posner MA, O'Connell JJ, Swain S, Mullins AN, Shwartz M, et al. Post-hospital medical respite care and hospital readmission of homeless persons. J Prev Interv Community. 2009;37(2):129-42.

51. Nordeck CD, Welsh C, Schwartz RP, Mitchell SG, Cohen A, O'Grady KE, et al. Rehospitalization and substance use disorder (SUD) treatment entry among patients seen by a hospital SUD consultation-liaison service. Drug Alcohol Depend. 2018;186:23-8.

52. Nilsson SF, Laursen TM, Hjorthøj C, Nordentoft M. Homelessness as a predictor of mortality: an 11-year register-based cohort study. Soc Psychiatry Psychiatr Epidemiol. 2018;53(1):63-75.

53. Coldwell CM, Bender WS. The effectiveness of assertive community treatment for homeless populations with severe mental illness: a metaanalysis. Am J Psychiatr. 2007;164(3):393-9.

54. Ackerson TH. Evaluating use of continuous treatment teams for persons with mental illness and substance abuse. Psychiatr Serv. 1995;46(7):689

55. Drake RE, McHugo GJ, Clark RE, Teague GB, Xie H, Miles K, et al. Assertive community treatment for patients with co-occurring severe mental illness and substance use disorder: A clinical trial. Am J Orthop. 1998;68(2):201.

56. Baxter AJ, Tweed EJ, Katikireddi SV, Thomson H. Effects of Housing First approaches on health and well-being of adults who are homeless or at risk of homelessness: systematic review and meta-analysis of randomised controlled trials. J Epidemiol Community Health. 2019;73(5):379-87.

57. Bottner R, Moriates C, Tirado C. The Role of Hospitalists in Treating Opioid Use Disorder. J Addict Med. 2020;14(2): 178.

58. Brothers TD, Fraser J, MacAdam E, Morgan B, Francheville J, Nidumolu A Cheung C, Hickcox S, Saunders D, O'Donnell T, Genge L, Webster D. Implementation and evaluation of a novel, unofficial, trainee-organized hospital addiction medicine consultation service. Subst Abus. 2021;42(4):433-437. https://doi.org/10.1080/08897077.2020.1856291.

59. Tierney HR, Rowe CL, Coffa DA, Sarnaik S, Coffin PO, Snyder HR Inpatient Opioid Use Disorder Treatment by Generalists is Associated With Linkage to Opioid Treatment Programs After Discharge. J Addict Med. 2021 https://doi.org/10.1097/ADM.0000000000000851.

60. Englander H, Priest KC, Snyder H, Martin M, Calcaterra S, Gregg J. A Call to Action: Hospitalists' Role in Addressing Substance Use Disorder. J Hosp Med. 2019;14:E1-E4.

61. Society of Hospital Medicine. New Buprenorphine Practice Guidelines: FAQs. 2021, https://www.hospitalmedicine.org/globalassets/practicemanagement/practice-management-pdf/x-waiver-faq.pdf. Accessed December $12,2021$. 
62. Fiscella K, Wakeman SE, Beletsky L. Buprenorphine deregulation and mainstreaming treatment for opioid use disorder: $\mathrm{X}$ the $\mathrm{X}$ waiver. JAMA Psychiatry. 2019;76(3):229-30.

63. Saloner B, Andraka Christou B, Gordon AJ, Stein BD. It will end in tiers: A strategy to include "dabblers" in the buprenorphine workforce after the X-waiver. Subst Abus. 2021;42(2):153-157. https://doi.org/10.1080/ 08897077.2021.1903659.

64. Frank JW, Wakeman SE, Gordon AJ. No end to the crisis without an end to the waiver. Taylor \& Francis; 2018.

65. U.S. Department of Health and Human Services [HHS] HHS Expands Access to Treatment for Opioid Use Disorder. 2021, https://www.hhs. gov/about/news/2021/01/14/hhs-expands-access-to-treatment-foropioid-usedisorder.html. Accessed December 10. 2021.
66. Kim JH, Fine DR, Li L, Kimmel SD, Ngo LH, Suzuki J, et al. Disparities in United States hospitalizations for serious infections in patients with and without opioid use disorder: A nationwide observational study. PLoS Med. 2020;17(8):e1003247.

67. Auerbach C, Silverstein LB. Qualitative data: An introduction to coding and analysis: NYU press; 2003.

68. McNeely J, Troxel AB, Kunins HV, Shelley D, Lee JD, Walley A, et al. Study protocol for a pragmatic trial of the Consult for Addiction Treatment and Care in Hospitals (CATCH) model for engaging patients in opioid use disorder treatment. Addict Sci Clin Pract. 2019;14(1):1-12.

Publisher's Note: Springer Nature remains neutral with regard to jurisdictional claims in published maps and institutional affiliations. 